\title{
Assessment of the degree of agreement between the judgment of tracheotomy score and the clinician: a retrospective analysis
}

\section{Atsushi Abe ( $\boldsymbol{D}$ atsushi.a@ekisai.or.jp )}

Nagoya Ekisaikai Hospital https://orcid.org/0000-0001-8215-2769

\section{Kenichi Kurita}

Aichi-gakuin University

Yu Ito

Nagoya Ekisaikai Hospital

Hiroki Hayashi

Nagoya Ekisaikai Hospital

Eri Umemura

Nagoya Saisyukan Hospital

Moriyasu Adachi

Shizuoka General Hospital

\section{Research article}

Keywords: tracheotomy, Airway management, Cameron score, Gupta score, surgeon's experience, $\mathrm{k}$ coefficient

Posted Date: January 14th, 2020

DOI: https://doi.org/10.21203/rs.2.20667/v1

License: (a) This work is licensed under a Creative Commons Attribution 4.0 International License. Read Full License 


\section{Abstract}

Background: In oral cancer surgery, the decision to tracheotomize is often determined based on the experience of the surgeon. Sometimes, tracheotomy may be performed for cases that may not necessarily require tracheotomy. For such cases, safety is achieved by airway management, but the patients are exposed to tracheotomy-related complications. Several evaluation methods have been reported to predict the need for selective tracheotomy in patients with oral cancer. In this study, we investigated the competency of clinical scoring systems in identifying patients who require tracheotomy among the oral malignancy cases treated with surgery in our department, and examined the degree of agreement between the surgeon's decision and the scores of various scoring systems.

Methods: This study was conducted on 110 patients with oral cancer who were treated with surgery under general anesthesia in the Department of Oral and Maxillofacial Surgery, Nagoya Ekisaikai Hospital, between January 2007 and April 2018. Among them, 67 patients (44 male and 23 female), who were managed by resection and reconstruction, were retrospectively analyzed. To derive the score, we evaluated the endpoint of these indices from clinical records and images. We divided the patients, based on the Cameron and Gupta scores, into two groups: tracheotomy and no tracheotomy groups, and evaluated the degree of agreement by calculating the $\mathrm{\kappa}$ coefficient.

Results: After the assessment, the $\mathrm{k}$ coefficients of the Gupta and Cameron scores were $0.61(95 \% \mathrm{Cl}, 0.4-$ $0.82)$ and $0.6(95 \% \mathrm{Cl}, 0.38-0.82)$, respectively. The clinical evaluation of the $\mathrm{K}$ coefficient indicated that the Gupta and Cameron scores agreed substantially.

Discussion: These score matched the decision of the surgeon and confirmed that it was able to be applied to the decision of the airway management. However, these values are affected by prevalence. When unilateral total neck dissection and resection of the primary lesion were performed, though it was high-risk, the score was low, and an evaluation contradicted it.

Conclusions: In this study, the Cameron and Gupta scores agreed with the decision of the experienced surgeon to some extent, and were confirmed to adapt to the clinical judgment.

\section{Background}

Surgical treatment of oral cancer varies greatly depending on individual cases wherein some may require local excision or reconstruction. Such surgical invasions pose a risk of various postoperative complications, including surgical wound infection, necrosis of reconstructed flaps, postoperative bleeding, and upper airway obstruction. Postoperative complications may prohibit healing, extend the length of hospital stays, and be life-threatening [1-5]. Notably, the causes of airway obstruction include postoperative hematoma, pharyngolaryngeal edema, and morphological changes of the airway; thus, appropriate airway management is required $[6,7]$. There are three methods of postoperative airway management: 1) extubation, 2) endotracheal intubation under sedation, and 3) tracheostomy. At present, tracheostomy or prolonged intubation remains the major modality for airway management for patients 
with oral and oropharyngeal cancers undergoing major surgery. However, no clear criteria currently exist for determining which method to select.

Therefore, the method is often determined based on the experience of surgeons, considering interinstitutional differences and patient characteristics. However, according to literature, tracheotomy is associated with reported complication rates of $8-45 \%$ [8]. Therefore, some studies have been conducted to predict appropriate airway management strategies [9-12].

However, these methods may not be applicable for all cases due to the disparities between institutions or differences in patient backgrounds. Enforcement of tracheotomy as an endpoint is determined by a physician. Therefore, it is necessary to examine the agreement between the inter-rater evaluations on the need for tracheotomy, and to further plan standardization of the evaluation process. However, such examinations are not conducted.

The purpose of this study was to calculate a kappa coefficient to examine the degree of agreement between the physician's subjective evaluation and tracheotomy score evaluations.

\section{Methods}

We performed a retrospective analysis of these variables for all patients who had resection and primary flap reconstruction in our department, and included 110 patients with oral cancer (76 males and 34 females) who were treated with surgical methods under general anesthesia at the Department of Oral and Maxillofacial Surgery, Nagoya Ekisaikai Hospital, between January 2007 and April 2018. The study subjects were patients who underwent either broad resection of the primary lesion, followed by epidermization or major composite resections with reconstructions. Cancer staging was performed based on inspection, contrast-enhanced computed tomography, magnetic resonance imaging, and positron emission tomography-computed tomography. Surgical procedures and methods for airway management for all patients were discussed and selected during a tumor conference at our department. The decision for postoperative airway management was made based on the operator's experience. Usually large tumors (T4), the mouth floor or posterior lesions on the tongue, and bilateral neck dissection were considered for elective tracheotomy. Various factors contributed to the need for tracheotomy in patients, ranging from the extent of surgical resection to 1) patient background; 2) resection procedure, inclusion/exclusion of neck dissection, and reconstruction procedure; 3 ) method of airway management; and 4) scoring based on previously reported indices [8-11]. The parameters of the indices were evaluated and scored based on the patients' medical and imaging records. Patients scored by the Cameron and Gupta Scores were divided into 2 groups: the group requiring tracheotomy (tracheotomy group) and the group not requiring tracheostomy (no tracheotomy group), and agreement with the actual performance or non-performance of tracheotomy was evaluated with the $\mathrm{k}$ coefficient.

The parameters were studied for their impact on contribution towards a need for tracheotomy in a patient. A tracheotomy score, which was adopted from the scoring system recommended by Cameron (2009), was used to evaluate the state of the patient's airway based on the type of operation. We 
analyzed an inter-rater agreement between the physician's decision on tracheotomy and the tracheotomy score's evaluation using a kappa statistic.

\section{Ethics}

This retrospective cohort study was approved by the Nagoya Ekisaikai Hospital Ethics Committee (approval number 2018-009). Written informed consent was obtained from all patients for publication of this research paper and any accompanying images. All procedures were performed in accordance with the ethical standards of the institutional and/or national research committee and in line with the 1964 Declaration of Helsinki. The study was conducted in accordance with the Strengthening the Reporting of Observational Studies in Epidemiology (STROBE) Statement: guidelines for reporting observational studies.

\section{Statistical analysis}

The kappa coefficient (к) was used to evaluate reliability among evaluators and to compare the different methods with regard to the number of canals identified. The $\kappa$ coefficient was used instead of the intraclass correlation coefficient for ordinal scale scores. The agreement between the surgeon's decision and airway management suggested by the scores was analyzed using the $\mathrm{k}$ coefficient. Statistical significance level was $<5 \%$. All statistical analyses were performed with EZR (Saitama Medical Center, Jichi Medical University, Saitama, Japan), which is a graphical user interface for R (The R Foundation for Statistical Computing, Vienna, Austria). More precisely, it is a modified version of $\mathrm{R}$ commander designed to add statistical functions frequently used in biostatistics [13].

\section{Results}

A total of 110 patient records were included in the study. Patient characteristics, including tumor sites, operative approaches, and postoperative courses, are shown in Table 1. Patients were aged between 42 and 88 years (mean age, $63.4 \pm 11.0$ ). Primary lesion excision only was performed for 24 patients; among these, extubation was performed in 21 patients, intubation under sedation was performed in 1 patient, and tracheotomy was performed in 2 patients. Furthermore, primary lesion excision and neck dissection were performed in 30 patients; among these, extubation was performed in 1 patient, intubation under sedation was performed in 8 patients, and tracheotomy was performed in 21 patients. As a result of metastasis, neck dissection only was performed in 13 patients; extubation was performed in all of these patients (Table 1). 
Table 1

Patient characteristics

\begin{tabular}{|c|c|c|c|c|}
\hline \multirow[t]{2}{*}{ Factor } & \multirow[t]{2}{*}{ Group } & \multicolumn{3}{|c|}{ Airway management } \\
\hline & & $\begin{array}{l}\text { Immediate } \\
\text { Extubation }\end{array}$ & $\begin{array}{l}\text { Overnight } \\
\text { intubation }\end{array}$ & Tracheostomy \\
\hline$n$ & & 35 & 9 & 23 \\
\hline Age & & $63.5 \pm 11.6$ & $64.1 \pm 9.3$ & $63.0 \pm 11.5$ \\
\hline \multirow[t]{2}{*}{ Sex } & Male & 26 & 2 & 16 \\
\hline & Female & 9 & 7 & 7 \\
\hline \multirow[t]{6}{*}{ Stage } & 1 & 7 & 0 & 0 \\
\hline & 2 & 15 & 0 & 0 \\
\hline & 3 & 7 & 7 & 15 \\
\hline & $4 a$ & 2 & 2 & 7 \\
\hline & $4 b$ & 0 & 0 & 1 \\
\hline & $\begin{array}{l}\text { Delayed cervical lymph node } \\
\text { metastasis }\end{array}$ & 4 & 0 & 0 \\
\hline \multirow{5}{*}{$\begin{array}{l}\text { Tumor } \\
\text { site }\end{array}$} & Maxilla & 4 & 1 & 0 \\
\hline & Buccal & 4 & 2 & 3 \\
\hline & Mandible & 6 & 2 & 5 \\
\hline & Floor of mouth & 3 & 1 & 2 \\
\hline & Tongue & 18 & 3 & 13 \\
\hline
\end{tabular}

Detailed results of scores, using the methods reported by Cameron [9] and Gupta [11], are shown in Fig. 1 and Table 2. The number of patients suggested to require tracheotomy was within the range of $0-7$ (Cameron Score) and 0-8 (Gupta Score). Scoring using the methods reported by Cameron and Gupta clearly indicates whether a patient requires tracheotomy. Tracheotomy was performed in 9 patients in the no tracheotomy group rated by the Cameron Score. The details were as follows: partial glossectomy and total neck dissection $(n=3)$, posterior partial glossectomy $(n=2)$, buccal mucosa and total neck dissection ( $n=2)$, marginal mandibulectomy $(n=1)$, and segmental mandibulectomy $(n=1)$. The details of the patients undergoing tracheotomy in the no tracheotomy group rated by the Gupta Score were as follows: partial glossectomy and total neck dissection $(n=3)$, posterior partial glossectomy $(n=2)$, hemiglossectomy, forearm flap reconstruction, and total neck dissection $(n=5)$, subtotal glossectomy, forearm flap reconstruction, and total neck dissection $(n=3)$, marginal mandibulectomy plate 
reconstruction $(n=1)$, total neck dissection and oral floor resection $(n=1)$, and total neck dissection $(n=$ 1). The details of the patients who underwent tracheotomy in the no tracheotomy group rated by the

Table 2

Evaluation of clinical scores

\begin{tabular}{|lll|}
\hline & Cameron & Gupta \\
\hline Suggested tracheostomy & 16 & 10 \\
\hline True positive & 14 & 9 \\
\hline False positive & 2 & 1 \\
\hline False negative & 9 & 14 \\
\hline True negative & 42 & 43 \\
\hline Sensitivity & 0.61 & 0.39 \\
\hline Specificity & 0.96 & 0.98 \\
\hline Positive predictive value & 0.88 & 0.9 \\
\hline Negative predictive value & 0.82 & 0.75 \\
\hline Likelihood ratio for positive results & 13.39 & 17.22 \\
\hline Likelihood ratio for negative results & 0.41 & 0.62 \\
\hline
\end{tabular}

Tracheotomy was not performed in 2 patients in the tracheotomy group rated by the Cameron Score. One was sedated and intubated for total neck dissection, buccal mucosal resection, and mandibular segmental resection; and the other was sedated and intubated for mandibular segmental resection, hard tissue reconstruction, and total neck dissection. One patient in the tracheotomy group rated by the Gupta Score did not undergo tracheostomy. This patient was intubated for hard tissue reconstruction, total neck dissection, and segmental mandibulectomy. Ten patients in the tracheotomy group rated by the Kruse Score did not undergo tracheostomy. The details are as follows: partial maxillectomy $(n=2)$, marginal mandibulectomy $(n=1)$, partial glossectomy $(n=4)$, resection of oral floor $(n=3)$, and unilateral neck dissection $(n=2)$. All of the patients underwent reconstruction by skin grafting.

\section{Discussion}

Airway obstruction after oral cancer surgery occurs as a result of a combination of multiple factors, such as large area excision of the mandible, tongue, and floor of the oral cavity; bilateral neck dissection; use of bulky reconstruction flaps; postoperative hematoma and pharyngolaryngeal edema; and relaxation of the tongue muscle $[14,15]$. To avoid critical situations occurring as a result of postoperative airway obstructions, pathophysiological observations, such as those based on tracheal tug, neck ultrasound, $\mathrm{SpO}_{2}$, and monitoring by a capnometer, are necessary. However, reliability pertaining to diagnosis is low 
with any of these approaches. Therefore, in situations where difficulties in postoperative airway management can be predicted, the breathing status of patients should be carefully monitored to detect any abnormalities at an early time, enabling performance of emergency measures. Furthermore, patients at risk of airway obstruction should be identified such that they can undergo continued intratracheal intubation or selective tracheotomy $[16,17]$. Typically, intratracheal intubation under sedation is used to maintain the airway between 24 and 48 hours after surgery. Selective tracheotomy is recommended in cases where intratracheal intubation must be maintained for more than 2 days. In a national survey in the UK, tracheotomy was selected for $69 \%$ (39/57) of patients who underwent free-flap head and neck reconstructive surgery [18]. However, complications, such as bleeding, occlusion, local infection, and pneumonia occur at a rate of $4-8 \%$ in tracheotomy [17-20]. These complications result in prolonged recovery of the patient and longer hospital stays. Appropriate strategies for airway management, therefore, remain controversial, and selective tracheotomy is determined based on the surgeon's experience, which often varies between individuals. Therefore, research to establish criteria for performing tracheotomy is required. There have been several reports regarding evaluation methods to predict the necessity for selective tracheotomy in patients with oral cancer [9-12]. However, these methods may not be applicable for all patients due to disparities among institutions or differences in patient backgrounds. In this study, we examined the level of agreement between the need for tracheotomy based on these evaluation methods and the surgeon's decision, as well as cases with a discrepancy between them.

Kruse et al. were the first to report a scoring system that can be used to determine the need for tracheotomy [9]. They scored and evaluated 5 components (tumor localization, tumor size, pathological chest X-ray findings, multimorbidity, and alcohol consumption) to predict the risk of postoperative respiratory failure in 928 patients. Kruse reported sensitivity as $97.6 \%$. Notably, the scoring system by Kruse et al. does not evaluate various surgical factors. The expected reason for this may be that surgery is often avoided for patients with suspected disease based on chest X-rays; moreover, alcohol consumption is subject to recall bias. Surgical procedures, such as neck dissection and flap reconstruction, are highly invasive procedures; thus, these often are factors contributing to avoidance of surgery in patients with systemic disease. Considering such patients, this scoring method is not suitable for predicting postoperative airway management after surgical procedures or in the presence of localized tumors in patients with systemic disease.

Cameron et al. also reported a method that can be used to identify patients who require tracheotomy. Patients $(n=148)$ were scored based on tumor location, resected area of the mandible, neck dissection procedures, and reconstruction procedures [10]. This scoring system was created by combining elements used in evaluation based on the same items.

Based on the analysis of 486 patients, Gupta et al. reported scoring based on small and large categories, including resection, reconstruction methods, previous experience of radiotherapy, and degree of mouth opening; these are factors that may obstruct airway management even before surgery [12]. Gupta et al. reported that the sensitivity of the clinical assessment scoring system for tracheotomy (CASST) was 95.5\%; selectivity was 99.5\%; positive predictive value (PPV) was $96.9 \%$; and negative predictive value 
(NPV) was 99.3\%. Sensitivity was low in our patients, although selectivity and PPV were greater than $90 \%$. In this system, scores are given for surgery (especially areas of resection) and reconstruction procedures. In terms of prediction of airway management associated with surgery, details of surgery are incorporated within this system in comparison with the other systems; therefore, this system is expected to be useful for surgeons.

Whether high sensitivity or high selectivity is required for these tests depends on the clinical state and study population. Since a number of analyses showed false-negative results depending on the criteria, scores became relatively low in partial glossectomy, as well as in cases where forearm flap reconstruction was performed with pull-through or supraomohyoid neck dissection. It is controversial as to whether to perform tracheotomy or to simply maintain intratracheal intubation under sedation in such cases. However, it is understood that tracheotomy is not performed on a large number of cases, despite high risks of postoperative airway obstruction. Careful postoperative monitoring and structures for managing emergencies are required for such cases to avoid malpractice. In addition to a system that can be used for distinguishing at-risk patients from those with false-negative results, it is important to combine several systems for evaluation. Similarly, false-positive diagnosis for tracheotomy must be avoided for patients who do not require this procedure. In this study, the $\mathrm{k}$ coefficients for the Cameron and Gupta Scores were 0.61 and 0.6 , respectively, indicating substantial agreement. The reason why the patients who needed tracheotomy based on the scores actually did not undergo tracheostomy was considered as follows: the Cameron and Gupta Scores were high in patients who underwent resection of the mandibular area and surrounding tissue (e.g. buccal mucosa or floor of mouth) and hard tissue reconstruction. However, in these cases, the surgeons decided that intubation under sedation was possible when postoperative aspiration was not a concern. We confirmed that the Cameron and Gupta Scores were consistent with the surgeon's decision to some extent and could be applied generally to clinical decisions in hospitals in Japan.

These scores are weighted for bilateral neck dissection. However, when unilateral total neck dissection was performed along with resection of the primary lesion, the score was not always high, which was considered to be the cause of discrepancy. These values are affected by prevalence, although scores were effective for screening postoperative airway management. However, Schmutz et al. have reported that patient population differs by institution; therefore, they failed to predict the need for tracheotomy based on these clinical scoring systems [21]. Similarly, Lee et al. reported that they could not identify correlations between the need for tracheotomy and the clinical findings for patients with oral cancer based on the Cameron score [22]. Moreover, Benetar et al. conducted analysis based on the Cameron score for performing elective tracheotomy in oral cancer surgery. Analysis revealed high selectivity and PPV (90\% for both), low sensitivity (70\%), and NPV (67\%); these findings made it difficult to determine whether tracheotomy was necessary [23]. Upon reviewing reports that re-evaluate these scores, patients who actually require tracheotomy may not be accurately identified, and tracheotomy is suggested in a large proportion of cases. This is likely due to large differences in choices of surgical methods, decisions based on surgeons' experience, and patient population. Moreover, postoperative hematoma and pharyngolaryngeal edema cannot be predicted directly from the scores investigated in this study. 
Clinically, airway obstruction rapidly advances in such cases, which then becomes irreversible. Intratracheal intubation or tracheotomy must be selected under emergency situations; these urgent decisions are the largest problem. These scoring systems were reported years ago, and since then, there have been advancement in equipment (e.g., energy devices), improvement in surgical techniques and perioperative care, and extensive changes in the applicability criteria of surgical procedures.

Establishment of a scoring system that accommodates such advancements and changes is required in the future.

There were a few limitations in this study. Notably, we excluded patients who underwent cerclage because the risk of postoperative airway obstruction was expected to be low in such cases. However, there have been reported cases of severe outcomes resulting from occlusions, even if resection was solely performed on the frontal part of the mandible, as well as in cases where only the primary lesion was excised or a single neck dissection was performed [22, 23]. Even in cases that appear to be low risk, resection or abrasion of the genioglossus muscle, geniohyoid muscle, or mylohyoid muscle may cause deterioration in airway obstruction due to the loss of support of the hyoid bone. Such procedures with moderate surgical invasion are managed outside the intensive care unit and, therefore, pose a risk of delayed treatment of the airway obstruction. Evaluations of such cases are required in future.

\section{Conclusions}

We examined the degree of agreement between the physician's evaluation and tracheotomy scores' evaluations on the need for tracheotomy. After the assessment, the к coefficients of the Gupta and Cameron scores were $0.61(95 \% \mathrm{Cl}, 0.4-0.82)$ and $0.6(95 \% \mathrm{Cl}, 0.38-0.82)$, respectively. As a result, moderate congruity was found between the physician's evaluation and the tracheotomy scores' evaluations. In this study, the Cameron and Gupta scores agreed with the surgeon's experienced judgment to some extent, and they were confirmed to be able to adapt to clinical judgment in the hospital setting. These values are affected by prevalence; however, the scores were effective for screening postoperative airway management. Importantly, postoperative hematoma and pharyngolaryngeal edema cannot be predicted directly from the scores investigated in this study; therefore, scoring systems that can accommodate such changes should be established.

\section{Abbreviations}

PPV, positive predictive value; NPV, negative predictive value; CASST, clinical assessment scoring system for tracheostomy; STROBES, Strengthening the Reporting of Observational Studies in Epidemiology Statement

\section{Declarations}

\section{Ethics approval and consent to participate}


The present retrospective cohort study was approved by the Nagoya Ekisaikai Hospital Institutional Review Board (no. 2018-009). Individual consent from each patient was not required because extubation after major oral cancer surgery is the standard approach in our institution. Since the study was retrospective, only closed cases were included, and the institutional review board waived the need for obtaining the consent of the retrospectively analyzed patients. All procedures were performed in accordance with the ethical standards of the institutional and/or national research committee and in line with the 1964 Declaration of Helsinki.

\section{Consent for publication}

Written informed consent was obtained from the patient for publication of this case report and any accompanying images.

\section{Availability of data and materials}

All data generated or analyzed during this study are included in this published article. The datasets used and/or analyzed during the current study are available from the corresponding author on reasonable request.

\section{Competing interests}

The authors declare that they have no competing interests.

\section{Funding}

The present research did not receive any specific grant from funding agencies in the public, commercial, or not-for-profit sectors.

\section{Authors' contributions}

AA conceived, designed, and coordinated the study; and wrote the manuscript. KK and EU critically revised the manuscript for important intellectual content and gave the final approval of the version to be submitted. $\mathrm{HH}, \mathrm{YI}$, and MA collected the clinical data and drafted the article. All authors read and approved the final manuscript. 


\section{Acknowledgements}

We would like to thank Editage (www.editage.com) for English language editing.

\section{References}

1. Bartella AK, Sander AK, Kamal M, Steegmann J, Kloss-Brandstätter A, Teichmann J, et al. Preoperative assessment of the risk of postoperative death in patients with oral squamous cell carcinoma: a consideration beyond age, sex, and stage of cancer. Br J Oral Maxillofac Surg. 2018;56:322.

2. Van Abel KM, Moore EJ. Surgical management of the base of tongue. Oper Tech Otolaryngol - Head Neck Surg. 2013;24:74.

3. Siddiqui A, Dogar S, Lal S, Akhtar S, Khan F. Airway management and postoperative length of hospital stay in patients undergoing head and neck cancer surgery. J Anaesthesiol Clin Pharmacol. 2016;32:49.

4. de Melo GM, Ribeiro KC, Kowalski LP, Deheinzelin D. Risk factors for postoperative complications in oral cancer and their prognostic implications. Arch Otolaryngol Head Neck Surg. 2001;127:828.

5. Charters P, Ahmad I, Patel A, Russell S. Anaesthesia for head and neck surgery: United Kingdom National Multidisciplinary Guidelines. J Laryngol Otol. 2016;130:S23.

6. Uehara M, Kokuryo S, Sasaguri M, Tominaga K. Emergency cricothyroidotomy for difficult airway management after asynchronous bilateral neck dissections: A case report and literature review. $\mathrm{J}$ Oral Maxillofac Surg. 2015;73:2066e1.

7. Quick E, Byard RW. Postoperative cervical soft tissue hemorrhage with acute upper airway obstruction. J Forensic Sci. 2013;58:S264.

8. Castling B, Telfer M, Avery BS. Complications of tracheostomy in major head and neck cancer surgery; a retrospective study of 60 consecutive cases. $\mathrm{Br} J$ Oral Maxillofac Surg. 1994;32:3-5.

9. Kruse-Lösler B, Langer E, Reich A, Joos U, Kleinheinz J. Core system for elective tracheotomy in major head and neck tumour surgery. Acta Anaesthesiol Scand. 2005;49:654.

10. Cameron M, Corner A, Diba A, Hankins M. Development of a tracheostomy scoring system to guide airway management after major head and neck surgery. Int J Oral Maxillofac Surg. 2009;38:846.

11. Kim YH, Kim MY, Kim CH. Elective tracheostomy scoring system for severe oral disease patients. J Korean Assoc Oral Maxillofac Surg. 2014;40:211.

12. Gupta K, Mandlik D, Patel D, Patel P, Shah B, Vijay DG, et al. Clinical assessment scoring system for tracheostomy (CASST) criterion: Objective criteria to predict pre-operatively the need for a tracheostomy in head and neck malignancies. J Craniomaxillofac Surg. 2016;44:1310. 
13. Kanda Y. Investigation of the freely available easy-to-use software 'EZR' for medical statistics. Bone Marrow Transplant. 2013;48(3):452-8.

14. Agnew J, Hains D, Rounsfell B. Management of the airway in oral and oropharyngeal resections. Aust N Z J Surg. 1992;62:652.

15. Bernard AC, Kenady DE. Conventional surgical tracheostomy as the preferred method of airway management. J Oral Maxillofac Surg. 1999;57:310.

16. Phero JC, Rosenberg MB, Giovannitti JA. Adult airway evaluation in oral surgery. Oral Maxillofac Surg Clin North Am. 2013;25:386.

17. Harréus U. Surgical errors and risks - the head and neck cancer patient. GMS Curr Top Otorhinolaryngol Head Neck Surg. 2013;12.

18. Marsh M, Elliott S, Anand R, Brennan PA. Early postoperative care for free flap head \& neck reconstructive surgery - a national survey of practice. Br J Oral Maxillofac Surg. 2009;47:182.

19. Delaney A, Bagshaw SM, Nalos M. Percutaneous dilatational tracheostomy versus surgical tracheostomy in critically ill patients: a systematic review and meta-analysis. Crit Care. 2006;10:R55.

20. Fattahi T, Vega L, Fernandes R, Goldman N, Steinberg B, Schare H. Our experience with 171 open tracheostomies. J Oral Maxillofac Surg. 2012;70:1699.

21. Schmutz A, Dieterich R, Kalbhenn J, Voss P, Loop T, Heinrich S. Protocol based evaluation for feasibility of extubation compared to clinical scoring systems after major oral cancer surgery safely reduces the need for tracheostomy: A retrospective cohort study. BMC Anesthesiol. 2018;18:1.

22. Lee HJ, Kim JW, Choi SY, Kim CS, Kwon TG, Paeng JY. The evaluation of a scoring system in airway management after oral cancer surgery. Maxillofac Plast Reconstr Surg. 2015;37:19.

23. Benatar-Haserfaty J, Picón-Molina M, Meléndez-Salinas DA, Palacios-López C. Usefulness of the Cameron tracheostomy scoring system after oral tumor surgery. Rev Esp Anestesiol Reanim. 2014;61:369.

\section{Figures}




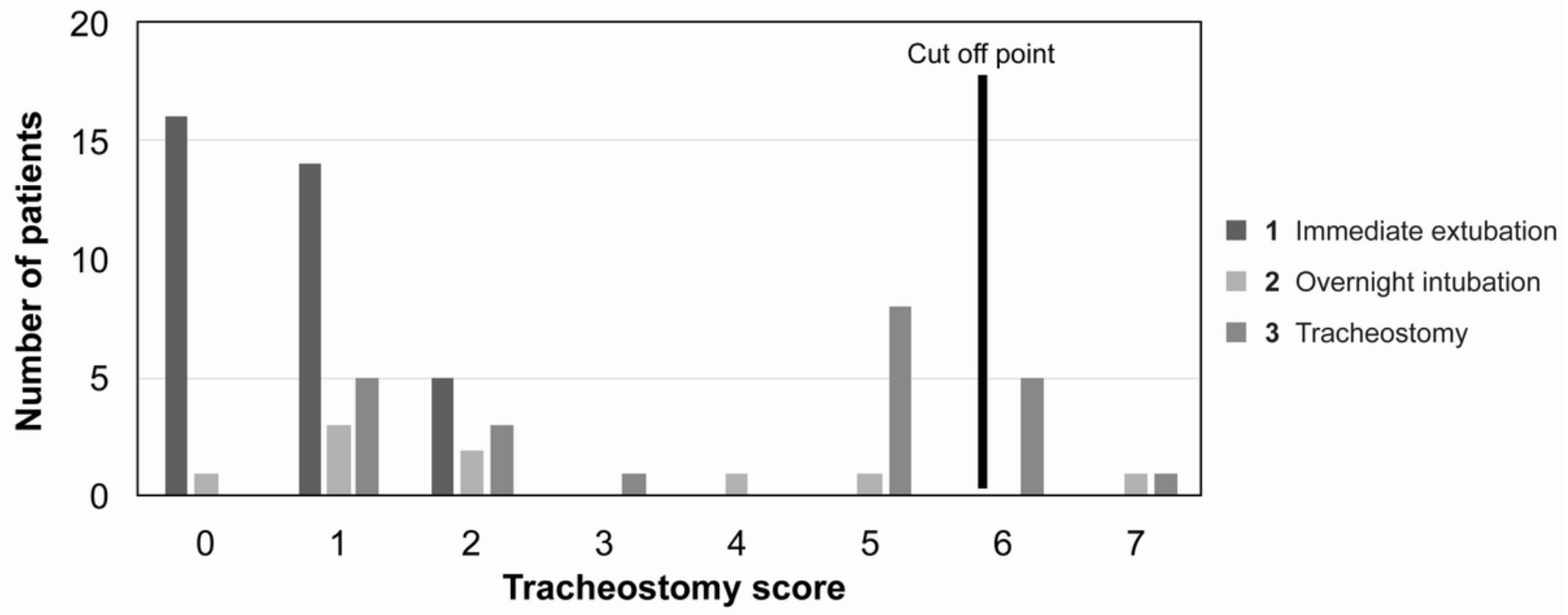

(a) Cameron score

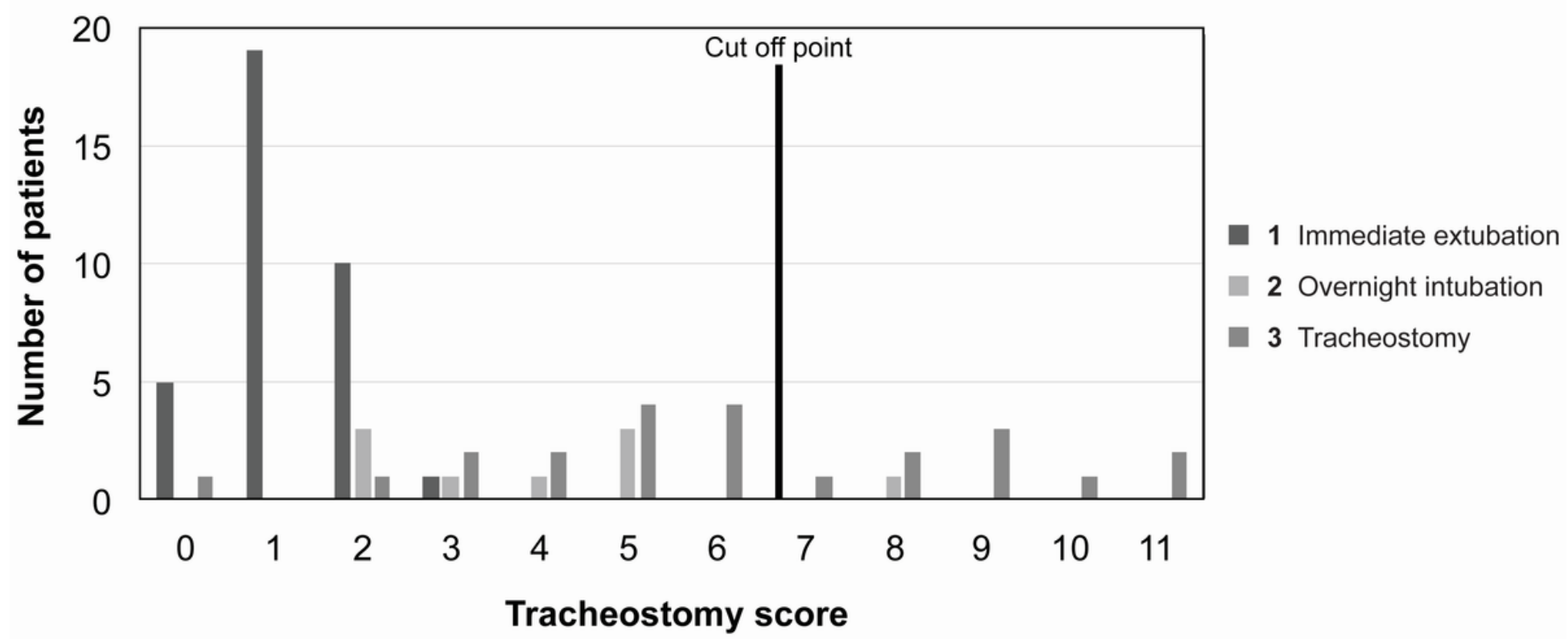

(b) Gupta score

Figure 1

Number of patients in the airway management group, according to tracheostomy score a: Cameron score b: Gupta score 